\title{
The Inhibitory Effect of Eupatilin on the Agonist-Induced Regulation of Vascular Contractility
}

\author{
Hyun Dong Je ${ }^{1, *}$, Hyeong-Dong $\mathrm{Kim}^{2, *}$, and Ji Hoon Jeong ${ }^{3,4}$ \\ ${ }^{1}$ Department of Pharmacology, College of Pharmacy, Catholic University of Daegu, Gyeongbuk 712-702, ${ }^{2}$ Department of Physical Therapy, \\ College of Health Science, Korea University, Seoul 136-701, ${ }^{3}$ Department of Pharmacology, College of Medicine, Chung-Ang University, \\ ${ }^{4}$ Research Institute for Translational System Biomics, Chung-Ang University, Seoul 156-756, Korea
}

\begin{abstract}
The present study was undertaken to investigate the influence of eupatilin on vascular smooth muscle contractility and to determine the mechanism involved. Denuded aortic rings from male rats were used and isometric contractions were recorded and combined with molecular experiments. Eupatilin more significantly relaxed fluoride-induced vascular contraction than thromboxane $A_{2}$ or phorbol ester-induced contraction suggesting as a possible anti-hypertensive on the agonist-induced vascular contraction regardless of endothelial nitric oxide synthesis. Furthermore, eupatilin significantly inhibited fluoride-induced increases in pMYPT1 levels. On the other hand, it didn't significantly inhibit phorbol ester-induced increases in pERK1/2 levels suggesting the mechanism involving the primarily inhibition of Rho-kinase activity and the subsequent phosphorylation of MYPT1. This study provides evidence regarding the mechanism underlying the relaxation effect of eupatilin on agonistinduced vascular contraction regardless of endothelial function.
\end{abstract}

Key Words: ERK1/2, Eupatilin, MYPT1, Rho-kinase, Vasodilation

\section{INTRODUCTION}

Artemisia asiatica Nakai (family Asteraceae) is an herbal medicine used as a hepatoprotective, antioxidative, anti-inflammatory, and antibacterial agent [1,2]. Eupatilin, a flavone or a type of flavonoid, is isolated from Artemisia asiatica Nakai and used in the treatment of acid-related disorders. We investigated the possible influence and related mechanisms of the anti-inflammatory eupatilin on vascular smooth muscle contractility to develop a better antihypertensive. Denuded aortic rings from male SpragueDawley rats were used and isometric contractions were recorded using a computerized data acquisition system. These data were combined with molecular experiments.

Alterations in the arterial tone are frequently associated with cardiovascular diseases constituting an important cause of morbidity and mortality in humans, one of which is hypertension that is a multifactorial disorder that involves many mechanisms including endothelial dysfunction

\footnotetext{
Received October 18, 2012, Revised January 10, 2013, Accepted January 19, 2013

Correspondence to: Ji Hoon Jeong, Department of Pharmacology, College of Medicine, Chung-Ang University, 221, Heuksuk-dong, Dongjak-gu, Seoul 156-756, Korea. (Tel) 82-2-820-5688, (Fax) 82-2826-8752, (E-mail) jhjeong3@cau.ac.kr

*The authors contributed equally to this paper.
}

(1) (8) This is an Open Access article distributed under the terms of the Creative Commons Attribution Non-Commercial License (http:/ Creative Commons Attribution Non-Commercial License (http:// use, distribution, and reproduction in any medium, provided the original work is properly cited. and leading to risk factors for cardiovascular diseases. Besides endothelial dysfunction, it is generally accepted that vascular smooth muscle contractility is predominantly controlled by $\mathrm{Ca}^{2+}$ signaling involving $\mathrm{Ca}^{2+}$ influx, release or sensitization and regulating a $\mathrm{Ca}^{2+}$-dependent increase in the phosphorylation of a $20 \mathrm{kDa}$ myosin light chain $\left(\mathrm{MLC}_{20}\right)$ [3]. The extent of $\mathrm{MLC}_{20}$ phosphorylation or force of contraction induced by agonist stimulation is usually higher than that caused by an increase in the cytosolic $\mathrm{Ca}^{2+}$ concentration referred to as $\mathrm{Ca}^{2+}$ sensitization [3]. Subsequent studies suggested that the inhibition of MLC phosphatase by Rho-kinase [4-7] or thin filament regulation including the activation of protein kinase $\mathrm{C}$ (PKC), mitogenactivated protein kinase kinases (MEK) and extracellular signal regulated kinase (ERK) 1/2, and phosphorylation of the actin binding protein caldesmon [8] may be major components of the pathway that facilitates in $\mathrm{Ca}^{2+}$ sensitization.

In various smooth muscles, fluoride, phorbol ester or thromboxane $\mathrm{A}_{2}$ mimetic has been shown to induce contractions, which may be due to primarily enhanced $\mathrm{Ca}^{2+}$ sensitivity or partially increased $\mathrm{Ca}^{2+}$ concentration only in thromboxane $\mathrm{A}_{2}$ mimetic. In particular, fluoride has been known to induce contractions in blood vessel preparations and to be a potent stimulator of $\mathrm{Gs}, \mathrm{Gi}, \mathrm{Gq}$ and transducin [9-11]. It is possible that the contractions induced by fluoride or thromboxane $\mathrm{A}_{2}$ mimetic involve the RhoA/Rho-kinase pathway [12]. However, it has not been reported as to

ABBREVIATIONS: ERK, extracellular signal regulated kinase; MLC, myosin light chain; PKC, protein kinase C; MEK, mitogen-activated protein kinase kinase; $\mathrm{MYPT}_{1}$, myosin phosphatase targeting subunit 1. 
whether this pathway is inhibited during eupatilin-induced vascular smooth muscle relaxation in aortic rings precontracted with Rho-kinase activator fluoride or phorbol ester. Therefore, the aim of the present study was to investigate the possible roles of Rho-kinase or MEK inhibition on $\mathrm{Ca}^{2+}$ desensitization during the eupatilin-induced relaxation of isolated rat aortas by using RhoA/Rho-kinase activators such as a full activator fluoride or thromboxane $\mathrm{A}_{2}$ or a partial activator phorbol ester excluding endothelial nitric oxide synthesis.

\section{METHODS}

\section{Tissue preparation}

Male Sprague-Dawley rats weighing 300 350 g were anesthetized with sodium pentobarbital (50 $\mathrm{mg} / \mathrm{kg}$ i.p.) as subjected to cervical dislocation, in accord with the procedures approved by the Institutional Animal Care and Use Committee at our institutions. Thoracic aortas were quickly removed and immersed in oxygenated $\left(95 \% \mathrm{O}_{2} / 5 \% \mathrm{CO}_{2}\right)$ physiological saline solution composed of $(\mathrm{mM}): 115.0 \mathrm{NaCl}$, $4.7 \mathrm{KCl}, 2.5 \mathrm{CaCl}_{2}, 1.2 \mathrm{MgCl}_{2}, 25.0 \mathrm{NaHCO}_{3}, 1.2 \mathrm{KH}_{2} \mathrm{PO}_{4}$, and 10.0 dextrose ( $\mathrm{pH} 7.4)$. They were then freed of all adherent connective tissue, and aortic endothelia were removed by gentle abrasion using a cell scraper.

\section{Contraction measurements}

Two stainless-steel triangles were inserted through each vessel ring and each aortic ring was then suspended in a water-jacketed organ bath $(10 \mathrm{ml})$ maintained at $37^{\circ} \mathrm{C}$ and aerated with a mixture of $95 \% \mathrm{O}_{2}$ and $5 \% \mathrm{CO}_{2}$. One triangle was anchored to a stationary support, and the other was connected to an isometric force transducer (Grass FT03C, Quincy, Mass., USA). The rings were stretched passively by applying an optimal resting tension of $2.0 \mathrm{~g}$, which was maintained throughout the experiment. Each ring was equilibrated in the organ bath solution for $60 \mathrm{~min}$ before contractile responses to $50 \mathrm{mM} \mathrm{KCl}$ were measured. Isometric contractions were recorded using a computerized data acquisition system (PowerLab/8SP, AD Instruments, Castle Hill, NSW, Australia).

The direct effect of eupatilin was determined by addition of it after $\mathrm{KCl}(50 \mathrm{mM})$, thromboxane $\mathrm{A}_{2}(0.1 \mu \mathrm{M})$, phorbol ester $(1 \mu \mathrm{M})$ or fluoride $(8 \mathrm{mM})$ induced contractions had plateaued in normal Krebs' solution.

\section{Western blot analysis}

Muscle strips were quick-frozen by immersion in a dry ice/acetone slurry containing $10 \%$ trichloroacetic acid (TCA) and $10 \mathrm{mM}$ dithiothreitol (DTT). Muscles were stored at $-80^{\circ} \mathrm{C}$ until use. Tissues were brought up to room temperature in a dry ice/acetone/ TCA/DTT mixture and then homogenized in a buffer containing $20 \mathrm{mM}$ MOPS, $4 \%$ SDS, $10 \%$ glycerol, $10 \mathrm{mM}$ DTT, $20 \mathrm{mM} \beta$-glycerophosphate, 5.5 $\mu \mathrm{M}$ leupeptin, $5.5 \mu \mathrm{M}$ pepstatin, $20 \mathrm{kIU}$ aprotinin, $2 \mathrm{mM}$ $\mathrm{Na}_{3} \mathrm{VO}_{4}, 1 \mathrm{mM} \mathrm{NaF}, 100 \mu \mathrm{M} \mathrm{ZnCl}, 20 \mu \mathrm{M}$ 4-(2-aminoethyl) benzenesulphonyl fluoride (AEBSF) and $5 \mathrm{mM}$ EGTA. Protein-matched samples (modified Lowry protein assay, DC Protein Assay Kit, Bio-Rad) were electrophoresed on sodium dodecyl sulfate polyacrylamide gel electrophoresis SDS-PAGE (Protogel, National Diagnostics), transferred to polyvinylidene fluoride PVDF membranes, and subjected to immunostaining and densitometry using appropriate antibodies. The success of protein matching was confirmed by Naphthol Blue Black staining of the membrane and by densitometry of the actin band. Lane loading variations were corrected by normalization versus $\beta$-actin. Sets of samples produced during individual experiments were run in the same gel and densitometry was performed on the same film.

\section{Chemicals and antibodies}

Drugs and chemicals were obtained from the following sources. Sodium fluoride, $\mathrm{KCl}$, acetylcholine, eupatilin, U46619 and phorbol 12,13-dibutyrate were purchased from Sigma (St. Louis, MO, USA). DTT, TCA and acetone were obtained from Fisher Scientific (Hampton, NH, USA). Enhanced chemiluminescence (ECL) kits were from Pierce (Rockford, IL, USA). Antibodies against phospho-myosin phosphatase targeting subunit protein 1 (phospho-MYPT1) at Thr855 (1:5,000), MYPT1, ERK or phosphoERK at Thr202/Tyr204 were purchased from Cell Signaling Technology (Danvers, MA, USA) or Upstate Biotechnology (Lake Placid, NY, USA) to determine levels of RhoA/ Rho-kinase activity [13,14] or MEK activity. Anti-mouse IgM (goat) and anti-rabbit IgG (goat), conjugated with horseradish peroxidase, were used as secondary antibodies $(1: 2,000$ and 1 : 2,000, respectively, Upstate, Lake Placid, NY). Eupatilin was prepared in dimethyl sulfoxide (DMSO) as a $100 \mathrm{mM}$ stock solution and frozen at $-20^{\circ} \mathrm{C}$ for later use. DMSO alone had no observable effect at concentrations used (data not shown).

\section{Statistics}

The data were expressed as mean \pm standard error of the mean (SEM). The student's unpaired $t$ test was used to determine the statistical significance of the means between two groups using SPSS 12.0 (SPSS Inc., Chicago, Illinois, U.S.A.). p-values $<0.05$ were regarded as statistically significant.

\section{RESULTS}

\section{Effect of eupatilin on contractions of endothelium- denuded aortas induced by a full RhoA/Rho-kinase activator fluoride or thromboxane $A_{2}$}

Endothelium was removed by gentle abrasion with a cell scraper to identify the direct effect of eupatilin on vascular smooth muscle. The absence of endothelium was confirmed by a lack of relaxation after treating precontracted ring segments with acetylcholine $(1 \mu \mathrm{M})$. Eupatilin showed no significant effect on basal tension (data not shown), but significantly inhibited the contraction induced by a full activator fluoride at a low concentration regardless of endothelial nitric oxide synthesis (Fig. 1, 2). This suggests that the relaxation mechanism of eupatilin might involve the inhibition of Rho-kinase activity in addition to endothelial nitric oxide synthesis and the subsequent activation of guanylyl cyclase. Coincidentally, eupatilin at the same concentration slightly inhibited thromboxane $\mathrm{A}_{2}$ mimetic U46619induced contraction (Fig. 1, 3) suggesting that thromboxane $\mathrm{A}_{2}$ mimetic acts differently from a full activator where 

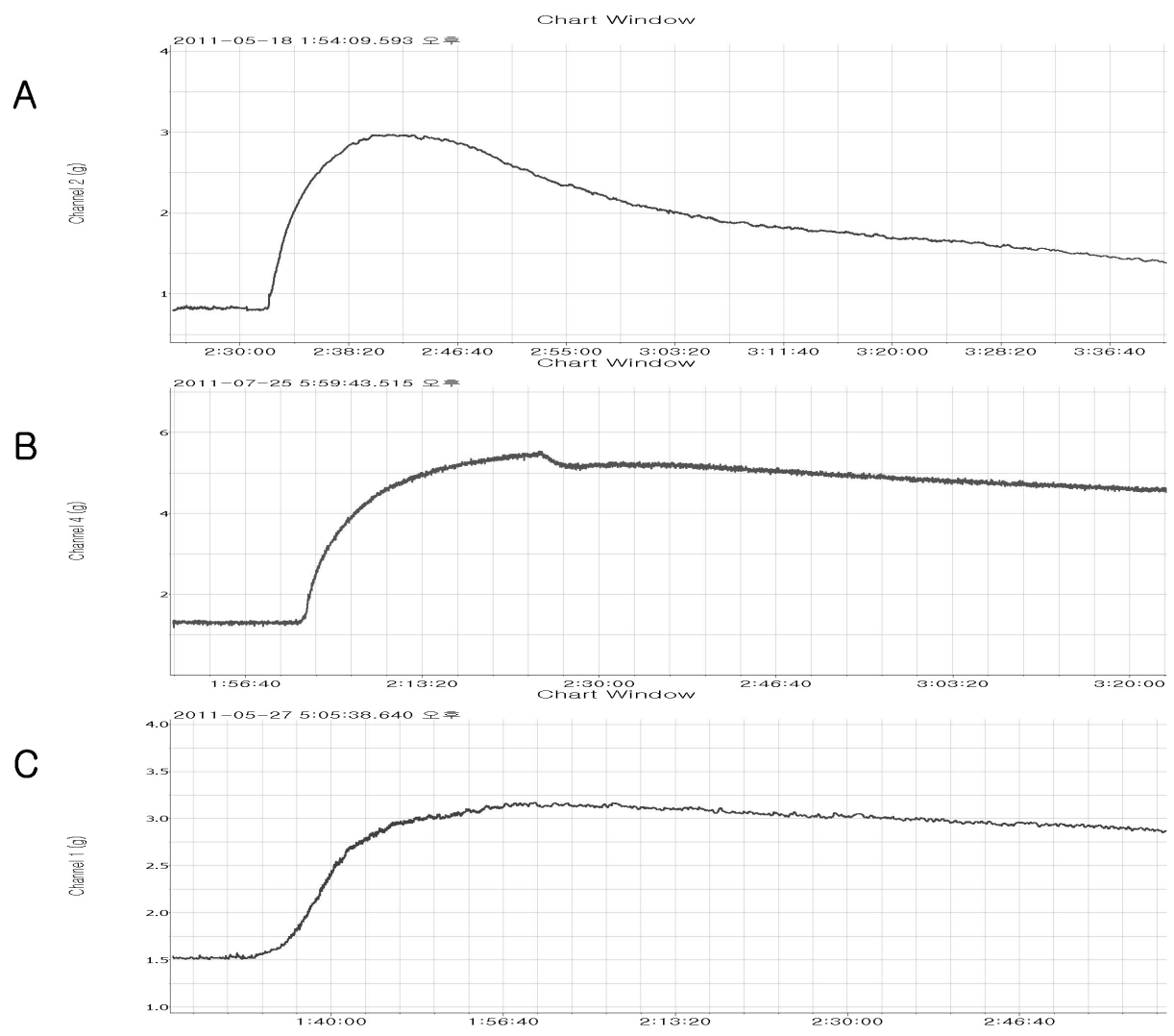

Fig. 1. A representative tracing of eupatilin-induced relaxation on the fluoride- (A), thromboxane A2- (B) or phorbol ester- (C) induced vasoconstriction in rat aorta.

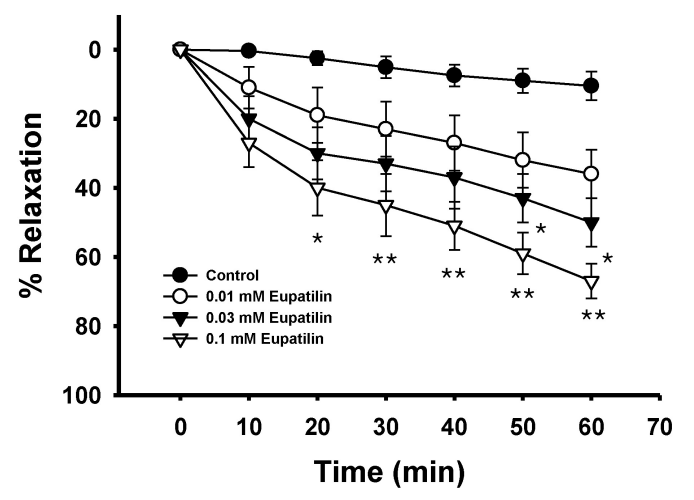

Fig. 2. Effect of eupatilin on fluoride-induced vascular contraction Each ring was equilibrated in the organ bath solution for $30 \sim 60$ min before relaxation responses to eupatilin were measured. Data are expressed as the means of $3 \sim 5$ experiments with vertical lines representing SEMs. Control respectively. ${ }^{*} \mathrm{p}<0.05,{ }^{* *} \mathrm{p}<0.01$ versus.

Rho-kinase activation was the main pathway.

\section{Effect of eupatilin on the contractions of denuded aortas induced by a partial RhoA/Rho-kinase activa- tor phorbol ester}

The vasoconstrictors used have been proved to be partial RhoA/Rho-kinase activators (data not shown). Interestingly, phorbol 12,13-dibutyrate-induced contraction was

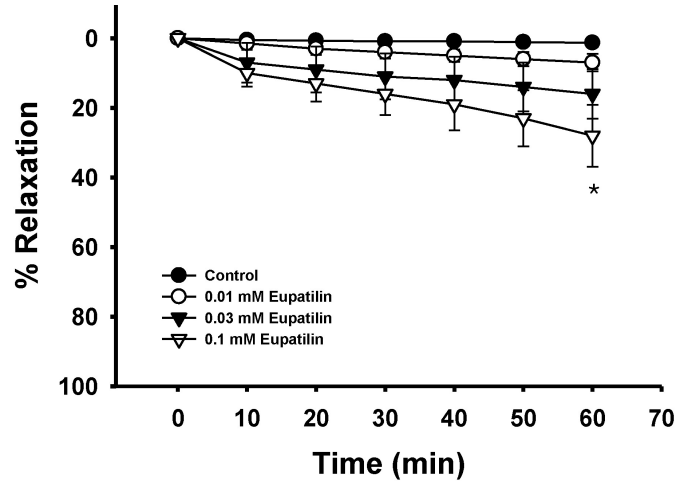

Fig. 3. Effect of eupatilin on thromboxane $\mathrm{A}_{2}$-induced vascular contraction. Each ring was equilibrated in the organ bath solution for $30 \sim 60 \mathrm{~min}$ before relaxation responses to eupatilin were measured. Data are expressed as the means of $3 \sim 5$ experiments with vertical lines representing SEMs. Control respectively. ${ }^{*} \mathrm{p}<$ 0.05 .

slightly inhibited by eupatilin at a high concentration regardless of endothelial nitric oxide synthesis (Fig. 1, 4), which suggested that other pathways including thin or actin filament regulation were slightly inhibited.

\section{Effect of eupatilin on the level of MYPT1 phospho- rylation at Thr- 855}

To confirm the role of eupatilin on the thick filament regulation of smooth muscle contractility, we measured levels 


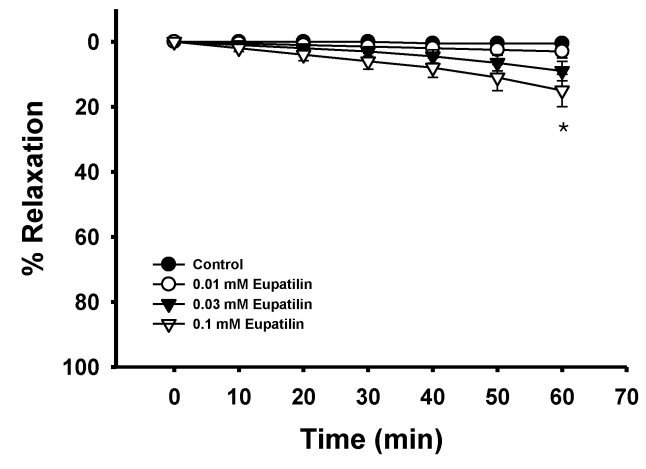

Fig. 4. Effect of eupatilin on phorbol ester-induced vascular contraction. Each ring was equilibrated in the organ bath solution for $30 \sim 60$ min before relaxation responses to eupatilin were measured. Data are expressed as the means of $3 \sim 5$ experiments with vertical lines representing SEMs. Control respectively. *p $<0.05$.

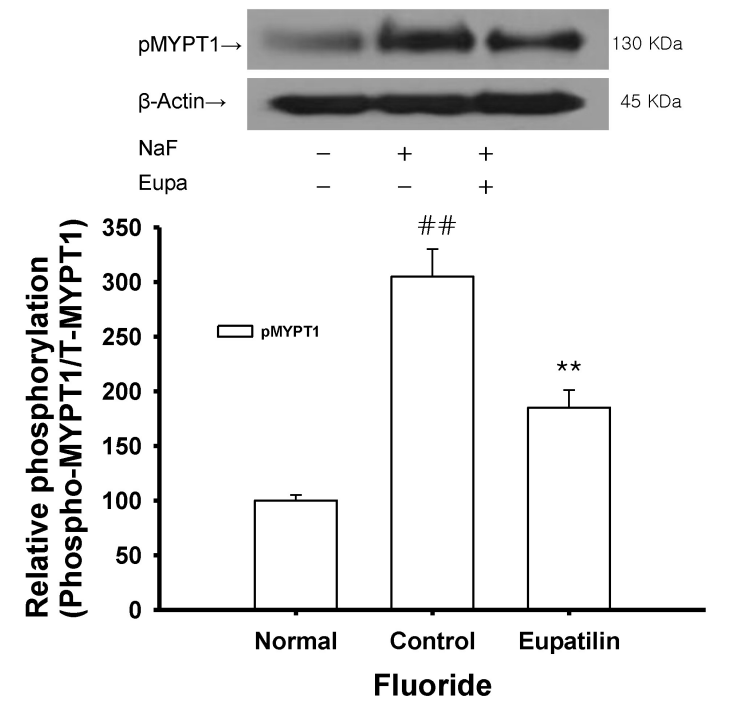

Fig. 5. Effect of eupatilin on fluoride-induced increases in phospho-MYPT1 levels. Phospho-MYPT1 protein levels were significantly decreased in quick frozen eupatilin-treated rat aorta in the absence of endothelium compared to vehicle-treated rat aorta precontracted with fluoride. The upper panel shows a typical blot and the lower panel shows average densitometry results for relative levels of phospho-MYPT1. Data are expressed as the means of $3 \sim 5$ experiments with vertical lines representing SEMs. ${ }^{* *} \mathrm{p}<0.01,{ }^{\# p}$ $<0.01$, versus control or normal group respectively. Eupa: $0.1 \mathrm{mM}$ eupatilin; NaF: $8 \mathrm{mM}$ sodium fluoride.

of myosin phosphatase targeting subunit 1 (MYPT1) and phospho-MYPT1 in muscles quick frozen after $60 \mathrm{~min}$ exposure to eupatilin for the equilibration. Each relaxing ring was precontracted with $8 \mathrm{mM}$ fluoride. This work was done using quick frozen eupatilin $(0.1 \mathrm{mM})$-treated rat aortas in the absence of endothelium and the levels were compared to those of vehicle-treated rat aortas (Fig. 5). Interestingly, a significant decrease in fluoride-induced MYPT1 phosphorylation at Thr855 was found to be led by eupatilin (Fig. 5). Thus, thick or myosin filament regulation including myosin phosphatase activation via RhoA/Rho-kinase inactivation might be involved in the reduced contractility of eu-

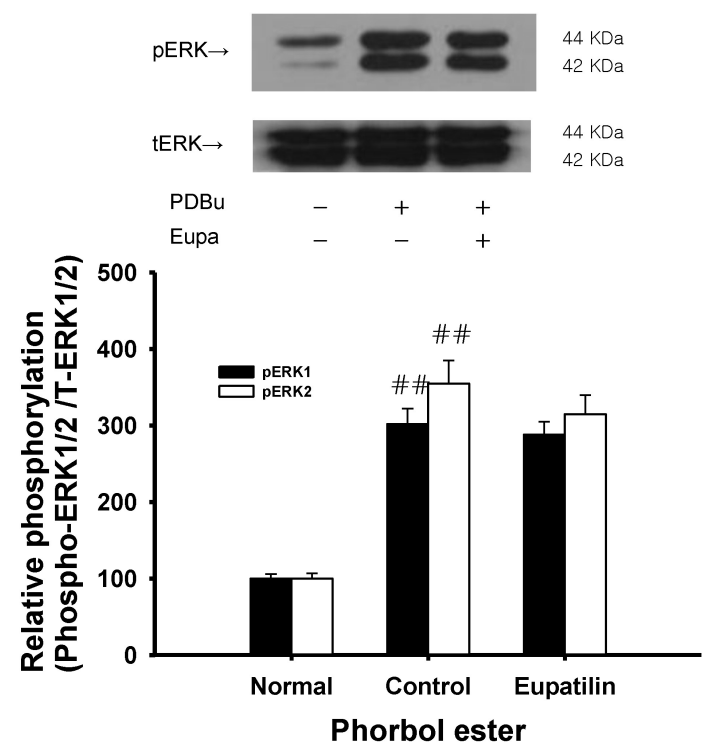

Fig. 6. Effect of eupatilin on phorbol ester-induced increases in phospho-ERK1/2 levels. Phospho-ERK1/2 protein levels were not decreased in quick frozen eupatilin-treated rat aortas in the absence of endothelium compared to vehicle-treated rat aortas precontracted with phorbol ester. The upper panel shows a typical blot and the lower panel shows average densitometry results for relative levels of phospho-ERK1/2. Data are expressed as the means of $3 \sim 5$ experiments with vertical lines representing SEMs. $\# \mathrm{p}<0.01$, versus normal group respectively. Eupa: $0.1 \mathrm{mM}$ eupatilin; PDBu: $1 \mu \mathrm{M}$ phorbol 12,13-dibutyrate.

patilin-treated rat aorta. Thus, the intensity of relaxation seems to directly proportional to the level of inhibition of MYPT1 phosphorylation by eupatilin.

\section{Effect of eupatilin on levels of ERK1/2 phosphory- lation at Thr-202/Tyr-204}

To confirm the role of eupatilin on thin filament regulation of smooth muscle contractility, we measured levels of ERK1/2 and phospho-ERK1/2 in muscles quick frozen after 60 minutes of exposure to eupatilin for the equilibration. Each relaxing ring was precontracted with $1 \mu \mathrm{M}$ phorbol ester (phorbol 12,13-dibutyrate). As compared with vehicle-treated rat aortas, no significant decrease in ERK 1/2 phosphorylation at Thr202/Tyr204 was led by eupatilin in these eupatilin $(0.1 \mathrm{mM})$-treated rat aortas in the absence of endothelium (Fig. 6) showing partial vasorelaxation (Fig. 4) and weak thin filament regulation. These findings show that thin or actin filament regulation including ERK1/2 phosphorylation via MEK activation might be of lesser importance in the decreased contractility induced by eupatilin at a low concentration $(0.03 \mathrm{mM})$.

\section{DISCUSSION}

The present study demonstrates that eupatilin can modulate the vascular contractility in an agonist-dependent manner. Interestingly, the mechanism involved seems to be not only endothelium-dependent but also to involve the inhibition of Rho-kinase and the partial inhibition of MEK activity. Eupatilin has been previously recognized for its 
anti-inflammatory activity. Therefore, we investigated whether the inhibition of RhoA/Rho-kinase or MEK activity contributes to eupatilin-induced vascular relaxation in rat aortas denuded and precontracted by a full RhoA/Rho-kinase activator fluoride or thromboxane $\mathrm{A}_{2}$ or by a partial activator phorbol ester.

The mechanism by which fluoride activates G-proteins has been established [9-11]. It has been reported that the effect of fluoride on heterotrimeric $G$ protein is due to the formation of $\mathrm{AlF}_{4}{ }^{-}$from fluoride and contamination of glassware [15,16], which mimics the effect of GTP [17]. Fluoride is also a classical Ser/Thr phosphatase inhibitor [18] and is routinely included in extraction buffers to prevent the dephosphorylations of proteins at Ser and Thr residues by endogenous phosphatases. On the other hand, previous studies that examined the mechanisms underlying arterial contractions induced by the phorbol ester or thromboxane $\mathrm{A}_{2}$ have reported variable findings with regard to the contraction triggered by Rho-kinase activation $[13,19]$. These findings are consistent with the notion that eupatilin can decrease fluoride, phorbol ester or thromboxane $\mathrm{A}_{2}$ induced contraction by inhibiting Rho-kinase activity.

The mechanisms by which Rho-kinase activation causes vascular contraction is an area of intense study, and several possibilities exist. For example, Rho-kinase phosphorylates myosin light chain phosphatase, which decreases phosphatase activity and causes a buildup of phosphorylated myosin light chains [20,21]. Rho-kinase has also been demonstrated to phosphorylate myosin light chains directly and independently of myosin light chain kinase and phosphatase activity [22]. Recently, Rho-kinase was found to be involved in vascular contractions evoked by fluoride, phorbol ester or thromboxane $\mathrm{A}_{2}[12,13,19]$.

The present study demonstrates that eupatilin ameliorates the maximal or submaximal contraction induced by vasoconstrictor fluoride, thromboxane $\mathrm{A}_{2}$ or phorbol ester endothelium-independently (Fig. 1 4), and that this ameliorative mechanism primarily involves the RhoA/Rho-kinase pathway. Previously, most vasodilation was believed to be caused by endothelial nitric oxide synthesis and the subsequent activation of guanylyl cyclase. In the present study, eupatilin at a low concentration significantly inhibited fluoride-induced contraction regardless of endothelial function (Fig. 2), but not thromboxane $\mathrm{A}_{2}$ - or phorbol ester-induced contraction (Fig. 3, 4). This suggests that the vascular contractions elicited by RhoA/Rho-kinase activators such as a full activator fluoride and a partial activator phorbol ester are achieved via different mechanisms (Fig. 5, 6). Therefore, we postulated that pathways other than the RhoA/ Rho-kinase pathway might be involved in $\mathrm{Ca}^{2+}$ sensitization induced by the phorbol ester. Thus, eupatilin at a low concentration might not inhibit $\mathrm{Ca}^{2+}$ mobilization $[23,24]$ or the phosphorylation of extracellular signal-regulated kinase (ERK), protein kinase C-potentiated inhibitory protein for protein phosphatase type 1 (CPI-17) or integrinlinked kinase (ILK) [25,26]. Furthermore, eupatilin decreased phosphorylation of MYPT1 at Thr855 induced by fluoride (Fig. 5), suggesting the inhibition of Rho-kinase activity as the major mechanism. However, eupatilin at the high concentration didn't significantly decrease the phosphorylation of ERK1/2 induced by phorbol ester (Fig. 6) with partial relaxation (Fig. 4) suggesting the inhibition of MEK activity as a minor mechanism.

In summary, eupatilin at a low concentration significantly attenuates the contractions induced by a full acti- vator fluoride regardless of endothelial function. In contrast, a partial activator phorbol ester-induced contraction was not significantly inhibited by eupatilin at this low concentration suggesting additional $\mathrm{Ca}^{2+}$ mobilization or the phosphorylations of ERK, CPI-17, ILK or ZIPK required for the partial activator-induced contractions. Thus, the mechanism underlying the relaxation induced by eupatilin at a low concentration in fluoride-induced contractions involves the inhibition of Rho-kinase activity and not the inhibition of MEK activity. Interestingly, during phorbol ester-induced contraction, no inhibition of MEK activity and subsequent ERK1/2 phosphorylation induced by eupatilin at a high concentration suggest that MEK activity is not importantly required for relaxation. In conclusion, in addition to endothelial nitric oxide synthesis, Rho-kinase inhibition makes a major contribution to the mechanism responsible for eupatilin-induced vasorelaxation in denuded muscle.

\section{ACKNOWLEDGEMENTS}

This work was supported by research grants from the Catholic University of Daegu in 2012.

\section{REFERENCES}

1. Kalemba D, Kusewicz D, Swiader K. Antimicrobial properties of the essential oil of Artemisia asiatica Nakai. Phytother Res. 2002;16:288-291.

2. Song HJ, Shin CY, Oh TY, Sohn UD. The protective effect ofeupatilin on indomethacin-induced cell damage in cultured feline ileal smooth muscle cells: involvement of HO-1 and ERK. J Ethnopharmacol. 2008;118:94-101.

3. Somlyo AP, Somlyo AV. Signal transduction and regulation in smooth muscle. Nature. 1994;372:231-236.

4. Somlyo AP, Somlyo AV. From pharmacomechanical coupling to G-proteins and myosin phosphatase. Acta Physiol Scand. 1998;164:437-448.

5. Uehata M, Ishizaki T, Satoh H, Ono T, Kawahara T, Morishita T, Tamakawa H, Yamagami K, Inui J, Maekawa M, Narumiya S. Calcium sensitization of smooth muscle mediated by a Rho-associated protein kinase in hypertension. Nature. 1997;389:990-994.

6. Sakurada S, Takuwa N, Sugimoto N, Wang Y, Seto M, Sasaki Y, Takuwa Y. $\mathrm{Ca}^{2+}$-dependent activation of Rho and Rho kinasein membrane depolarization-induced and receptor stimulation-induced vascular smooth muscle contraction. Circ Res. 2003;93:548-556.

7. Kitazawa T, Masuo M, Somlyo AP. G protein-mediated inhibition of myosin light-chain phosphatase invascular smooth muscle. Proc Natl Acad Sci USA. 1991;88:9307-9310.

8. Wier WG, Morgan KG. Alpha1-adrenergic signaling mechanisms in contraction of resistance arteries. Rev Physiol Biochem Pharmacol. 2003;150:91-139.

9. Kanaho Y, Moss J, Vaughan M. Mechanism of inhibition of transducin GTPase activity by fluoride and aluminum. J Biol Chem. 1985;260:11493-11497.

10. Blackmore PF, Exton JH. Studies on the hepatic calciummobilizing activity of aluminum fluoride and glucagon. Modulation by cAMP and phorbol myristate acetate. J Biol Chem. 1986;261:11056-11063.

11. Cockcroft S, Taylor JA. Fluoroaluminates mimic guanosine 5 '-[gamma-thio]triphosphate in activating the polyphosphoinositide phosphodiesterase of hepatocyte membranes. Role for the guanine nucleotide regulatory protein $\mathrm{Gp}$ in signal transduction. Biochem J. 1987;241:409-414.

12. Jeon SB, Jin F, Kim J, Kim SH, Suk K, Chae SC, Jun JE, Park WH, Kim IK. A role for Rho kinase in vascular contraction 
evoked by sodium fluoride. Biochem Biophys Res Commun. 2006;343:27-33.

13. Wilson DP, Susnjar M, Kiss E, Sutherland C, Walsh MP. Thromboxane $\mathrm{A}_{2}$-induced contraction of rat caudal arterial smooth muscle involves activation of $\mathrm{Ca}^{2+}$ entry and $\mathrm{Ca}^{2+}$ sensitization: Rho-associated kinase-mediated phosphorylation of MYPT1 at Thr-855, but not Thr-697. Biochem J. 2005;389: 763-774.

14. Wooldridge AA, MacDonald JA, Erdodi F, Ma C, Borman MA, Hartshorne DJ, Haystead TA. Smooth muscle phosphatase is regulated in vivo by exclusion of phosphorylation of threonine 696 of MYPT1 by phosphorylation of Serine 695 in response to cyclic nucleotides. J Biol Chem. 2004:279:34496-34504.

15. Zeng YY, Benishin CG, Pang PK. Guanine nucleotide binding proteins may modulate gating of calcium channels in vascular smooth muscle. I. Studies with fluoride. J Pharmacol Exp Ther. 1989;250:343-351.

16. Chabre M. Aluminofluoride and beryllofluoride complexes: a new phosphate analogs in enzymology. Trends Biochem Sci. 1990;15:6-10.

17. Bigay J, Deterre P, Pfister C, Chabre M. Fluoroaluminates activate transducin-GDP by mimicking the gamma-phosphate of GTP in its binding site. FEBS Lett. 1985;191:181-185.

18. Shenolikar S, Nairn AC. Protein phosphatases: recent progress. Adv Second Messenger Phosphoprotein Res. 1991;23:1-121.

19. Tsai MH, Jiang MJ. Rho-kinase-mediated regulation of receptor-agonist-stimulated smooth muscle contraction. Pflugers Arch. 2006;453:223-232.

20. Somlyo AP, Somlyo AV. Signal transduction by G-proteins, rho-kinase and protein phosphatase to smooth muscle and non-muscle myosin II. $J$ Physiol. 2000;522:177-185.

21. Pfitzer G. Invited review: regulation of myosin phosphorylation in smooth muscle. J Appl Physiol. 2001;91:497-503.

22. Amano M, Ito M, Kimura K, Fukata Y, Chihara K, Nakano T, Matsuura Y, Kaibuchi K. Phosphorylation and activation of myosin by Rho-associated kinase (Rho-kinase). J Biol Chem. 1996;271:20246-20249.

23. Davis MJ, Wu X, Nurkiewicz TR, Kawasaki J, Gui P, Hill MA, Wilson E. Regulation of ion channels by protein tyrosine phosphorylation. Am J Physiol Heart Circ Physiol. 2001;281: H1835-1862.

24. Low AM. Role of tyrosine kinase on $\mathrm{Ca}^{2+}$ entry and refilling of agonist-sensitive $\mathrm{Ca}^{2+}$ stores in vascular smooth muscles. Can J Physiol Pharmacol. 1996;74:298-304.

25. Deng JT, Van Lierop JE, Sutherland C, Walsh MP. $\mathrm{Ca}^{2+}$. independent smooth muscle contraction. a novel function for integrin-linked kinase. J Biol Chem. 2001;276:16365-16373.

26. Murányi A, MacDonald JA, Deng JT, Wilson DP, Haystead TA, Walsh MP, Erdodi F, Kiss E, Wu Y, Hartshorne DJ. Phosphorylation of the myosin phosphatase target subunit by integrin-linked kinase. Biochem J. 2002;366:211-216. 